\title{
Actitudes hacia el machismo y regulación emocional en estudiantes universitarios de Lima Metropolitana
}

\author{
Attitudes towards machismo and emotional regulation in university students \\ of Metropolitan Lima
}

Rosa Elena Huerta Rosales ${ }^{1}$, Renato Willy Santibáñez Olulo ${ }^{2}$, Natalia Luisa Ramírez Sáenz ${ }^{3}$, José Manuel Vega Gonzales ${ }^{4}$, Juan José Quispe Gamarra ${ }^{5}$, Samira Jasmín Terrel Quiquia ${ }^{6}$ y Sandra Karina Gamboa Peralta ${ }^{7}$ Universidad Nacional Mayor de San Marcos

\author{
Juan Carlos Escudero Nolasco ${ }^{8}$ \\ Universidad Privada del Norte y en \\ la Universidad César Vallejo
}

\section{Giovanni Chumbes Tellez ${ }^{9}$ CLINICAL HOME}

\author{
Judith Lila Potenciano Machado ${ }^{12}$
}

Sofía Guadalupe Merino Romero ${ }^{11}$ INNOA consulting and psichotherapy solutions

Adriana Fabiola Ortiz Mogollón ${ }^{13}$ Instituto para el Desarrollo infantil - Arie

\footnotetext{
1 Autor para correspondencia: rhuertar@unmsm.edu.pe ORCID: https://orcid.org/0000-0002-9197-6249

2 E-mail: rsantivanezo@unmsm.edu.pe ORCID: https://orcid.org/0000-0001-6835-6933

3 E-mail: nramirezs@unmsm.edu.pe ORCID: https://orcid.org/0000-0002-4837-7929

4 E-mail: jvegag@unmsm.edu.pe ORCID: https://orcid.org/0000-0002-4823-1997

5 E-mail: jose.quis.g@gmail.com ORCID: https://orcid.org/0000-0002-3454-8686

6 E-mail: samira.terrel@unmsm.edu.pe ORCID: https://orcid.org/0000-0001-7948-0382

7 E-mail: sandra.gamboa@unmsm.edu.pe ORCID: https://orcid.org/0000-0002-7605-6217

8 E-mail: juancescuderon@gmail.com ORCID: https://orcid.org/0000-0002-5158-7644

9 E-mail: giovannichumbest@gmail.com ORCID: https://orcid.org/0000-0002-7334-4029

10E-mail: anajulia.gonzales@unmsm.edu.pe ORCID: https://orcid.org/0000-0002-4083-2368

11 E-mail: sofiamerino09@gmail.com ORCID: https://orcid.org/0000-0001-7988-4782

12E-mail: lpotenciano12@gmail.com ORCID: https://orcid.org/0000-0001-7106-3437

13E-mail: adrianaortizm5@gmail.com ORCID: https://orcid.org/0000-0002-4581-1283
}

(C) Los autores. Este artículo es publicado por la Revista de Investigación en Psicología de la Facultad de Psicología, Universidad Nacional Mayor de San Marcos. Este es un artículo de acceso abierto, distribuido bajo los términos de la licencia Creative Commons Atribución 4.0 Internacional (CC BY 4.0) [https://creativecommons.org/licenses/by/4.0/deed.es] que permite el uso, distribución y reproducción en cualquier medio, siempre que la obra original sea debidamente citada de su fuente original. 


\title{
Resumen
}

El estudio analizó la relación entre las actitudes hacia el machismo y la regulación emocional, según universidad pública y privada y sexo; en una muestra de 830 estudiantes, 405 hombres (48.8\%) y 425 mujeres (51.2\%), con edades entre los 18 y 32 años, los que fueron examinados con una ficha de datos, la Escala de Evaluación de Actitudes hacia el machismo y el Cuestionario de regulación emocional. Se encuentra relación positiva y estadísticamente significativa entre la estrategia reevaluación cognitiva con el rechazo al machismo total y relación inversa con supresión y las seis áreas del machismo. Asimismo, existe asociación, en las mujeres, entre el rechazo al machismo con reevaluación cognitiva e inversa y significativa con la supresión en ambos sexos. Los de universidad pública obtienen una correlación estadísticamente significativa y positiva entre reevaluación cognitiva y rechazo al machismo global. Los de universidad privada muestran mayor rechazo al machismo . La reestructuración del significado disminuye los efectos del estrés. Si bien la supresión modifica la expresión de la respuesta emocional pero no altera la naturaleza de la emoción negativa dejando latente la propensión al machismo y a violencia de género común a todas las clases sociales y culturas.

Palabras clave: Actitudes hacia el machismo; regulación emocional; universitarios.

\begin{abstract}
The study analyzed the relationship between attitudes towards machismo and emotional regulation, according to public and private university and sex; In a sample of 830 students, 405 men (48.8\%) and 425 women (51.2\%), aged between 18 and 32 years, who were examined with a data sheet, the Scale of Evaluation of Attitudes towards machismo and the Emotional Regulation Questionnaire. A positive and statistically significant relationship is found between the cognitive reappraisal strategy with the rejection of total machismo and an inverse relationship with suppression and the six areas of machismo. Likewise, there is an association, in women, between the rejection of machismo with cognitive and inverse and significant reevaluation with the suppression in both sexes. Those from public universities obtain a statistically significant and positive correlation between cognitive reappraisal and rejection of global machismo. Those from private universities show greater rejection of machismo. The restructuring of meaning lessens the effects of stress. Although suppression modifies the expression of the emotional response but does not alter the nature of the negative emotion, leaving latent the propensity for machismo and gender violence common to all social classes and cultures.
\end{abstract}

Keywords: Attitudes towards machismo; emotional regulation; university students. 
Un problema psicosocial álgido en el país es la violencia de género, según la Encuesta Demográfica y de Salud Familiar (Instituto Nacional de Estadística e Informática [INEI], 2018), en donde las estadísticas describen que el $63,2 \%$ de las mujeres de 15 a 49 años, fueron víctimas de violencia, alguna vez por el esposo o compañero, donde la violencia psicológica $(58,9 \%)$, es la más elevada a través del control de la mujer. Problemática relacionada con la concepción social y cultural de las personas (Sanmartín, 2006; Gómez, 2007), agravada por los patrones culturales e idiosincráticos insertos en la forma de relación en la familia y expresadas a través de actitudes machistas, las que son aprendidas y modeladas desde la infancia. Para Castañeda (2009) todas las personas están implicadas en este fenómeno, hombres y mujeres, sin percatarse de ello.

El machismo es concebido como aquellas creencias, actitudes y conductas que sustentan, no solo una diferencia entre lo masculino y lo femenino, sino que los excluyen; así como la superioridad de lo masculino en las áreas que los hombres lo consideren (Castañeda, 2007). Expresadas en el presente de manera sutil, enmascarado e instituido culturalmente cómo micromachismos (Bonino, 2004) o machismo invisible (Castañeda, 2009), que no dejan de ser violentos sino más aceptadas y justificadas socialmente.

Oblitas (2009) declaró que la familia se sigue rigiendo por una cultura patriarcal como constructo social y cultural, en la que hombres y mujeres manifiestan conductas, hábitos y opiniones afines con lo establecido por la sociedad; revelando además que la violencia contra la mujer está unida al fenómeno del machismo, donde los hombres presentan un alto nivel de actitudes hacia el machismo en contraste con las mujeres.

La violencia de género provoca graves daños, tanto para la salud física como la psicológica (Calvo y Camacho, 2014; Samaniego y Freixas, 2010), tanto en hombres como en mujeres. En lo que respecta a las adolescentes, víctimas de maltrato en la relación de pareja, existe mayor riesgo de presentar trastornos conductuales y psicopatológicas asociados a la escasa habilidad para regular tanto las emociones positivas como negativas; según Patterson \& Sanson (1999, citado en Esteves y Jiménez, 2017) la agresión en niños y adolescentes influye en la pobre regulación de las propias emociones en particular para presentar problemas externalizantes en general.

Las emociones desempeñan importantes funciones adaptativas, pero, bajo determinados escenarios, las emociones negativas pueden dar lugar a desordenes, especialmente si esta deviene de patrones de comportamiento machista. Así, emociones negativas de ira y temor implican cambios psicofisiológicos y provocan en el organismo impulsos para la lucha o la huida. Lo cual generan disfunciones en las interacciones sociales, pudiendo perdurar la reactividad psicofisiológica y desencadenar comportamiento destructivo hacia uno mismo y los demás (Garland, Fredrickson, Kring, Johnson, Meyer y Penn, 2010; citados en Souto, 2013). 
La regulación de la emoción viene a ser un conjunto de procesos a través de los cuales las personas influyen en sus propias emociones y en las formas en que esas emociones se experimentan y expresan (Gross y Thompson, 2007). A su vez, estos procesos pueden ser conscientes o inconscientes, automáticos o intencionales, y su propósito puede ser disminuir, acentuar o solo el mantenimiento de las emociones de la persona (Gross y Thompson, 2007). Las dos estrategias según esta perspectiva son; la reevaluación y la supresión.

La reevaluación referida a la reestructuración del significado de una posible situación emocional, con menor contenido emocional (reevaluación cognitiva) se centra en los procesos antecedentes de la respuesta emocional, disminuyendo la presentación del estrés (Gross, 1998; Gross \& John, 2003; Gross \& Thompson 2007). La supresión, conlleva a la ocultación de las reacciones emocionales mediante respuestas conductuales más positivas; modula la respuesta de la persona debido a que inhibe la expresión de la respuesta emocional (Gross \& John, 2003), si bien lo modifica, aunque no reduce el estrés.

La regulación emocional tiene efecto protector frente a la aparición de respuestas físicas y psicológicas de estrés; constituyéndose en mediador de los recursos emocionales sobre las respuestas de tipo cognitivo (pensamientos negativos) y emocional (ira) (Souto, 2013).

Por otro lado, al ser la etapa universitaria un periodo crítico en el desarrollo de recursos de afrontamiento, según Brougham, Zail, Mendoza y Miller (2009), la universidad debe asumir la formación de habilidades de regulación del estrés, pues su carencia, se asocia con mengua en el bienestar físico, psicológico y social. Park et al., (2012, citado en Souto, 2013) consideraron esta etapa como esencial para formar relaciones de pareja y enfrentar las demandas de la sociedad respecto a los roles de género. Así, estudiantes con habilidades de regulación emocional, poseen mejores relaciones interpersonales y tendencia prosocial (Lopes, Salovey, Côté, Beers y Petty, 2005), para Winters, Clift \& Dutton (2004) la baja inteligencia emocional parece asociarse con violencia hacia la pareja.

Lo expuesto nos muestra que existe diferencia, aceptada como natural, entre hombres y mujeres con respecto a las emociones, (Giraldo, 1972), así como, algunas situaciones que se dan en la vida diaria, como el machismo, se van naturalizado en la población de ambos sexos.

A partir de lo planteado se consideró como objetivos, analizar la relación entre las actitudes hacia el machismo con las estrategias de regulación emocional en estudiantes universitarios, según universidad de procedencia (Pública y Privada) de Lima Metropolitana. Así mismo evidenciar las diferencias entre las actitudes hacia el machismo con las estrategias de regulación emocional según universidad de procedencia, como también el sexo. 


\section{MÉTODO}

Tipo y diseño de investigación: es un estudio empírico de estrategia asociativa de tipo predictivo con diseño correlacional simple, dado que para el análisis estadístico de la asociación entre variables utilizó un coeficiente de correlación apropiado a la naturaleza métrica de las variables (Ato et al., 2013).

\section{Participantes}

La muestra quedo compuesta por 830 estudiantes universitarios, se aplicaron un total de 850 de los cuales se eliminaron 15 por estar incompletos los cuestionarios; en cuanto al sexo masculino fueron 405 hombres $(48,8 \%)$ y 425 mujeres (51,2\%), del sexto al octavo ciclo. Asimismo, fueron 603 (72,7\%) de universidades públicas y 227 de privadas (27,3). En lo referente a la edad la mayormente tienen 21 años (24.8\%), seguidos de los de 20 años (19.2\%) y de 22 años (17.1\%). Por nivel socioeconómico principalmente pertenecen al nivel C $(61.3 \%$,) seguido del D $(16.7 \%)$ y en menor porcentaje los de nivel A (1.9) \%.

\section{Instrumentos}

Ficha de datos sociodemográficos: Elaborada por el investigador. Aplicada de manera agrupada. Permite recoger características relevantes de los sujetos estudiados como, edad, sexo, procedencia, nivel socioeconómico.

Para evaluar las actitudes hacia el machismo se utilizó la Escala de Evaluación de las Actitudes hacia el machismo elaborada en el Perú por Bustamante (1999), con cinco áreas; actitudes frente al dominio masculino, a la superioridad masculina, hacia la dirección del hogar, a la socialización del rol sexual masculino y femenino y al control de la sexualidad ejercida por los varones. Bustamante analizó la validez basada en el contenido y obtuvo valores de confiabilidad por consistencia interna mediante el coeficiente que oscilaron entre .83 a .89 en las cinco dimensiones.

En este estudio, para la Escala de actitudes hacia el machismo: se realizaron el análisis factorial exploratorio y confirmatorio, entendidos no como categorías cualitativamente distintas, sino como dos polos de un continuo (Ferrando y Anguiano-Carrasco, 2010), encontrando seis factores (dominio masculino, superioridad masculina, socialización del rol sexual masculino y femenino, control de la sexualidad ejercida por los varones, dirección del hogar y papel paterno); de 59 ítems planteados por la autora, se retiraron 18, quedando la Escala final con 41 items. En todas las cargas obtuvieron valores mayores a 40 .

La confiabilidad se evaluó mediante consistencia interna por medio del coeficiente omega, puesto que al trabajar con las cargas factoriales hace más estables 
los cálculos, reflejando la fiabilidad verdadera, además su valor no depende del número de ítems (Ventura y Caycho, 2017). Los valores en todas las variables superan el 0,70 siendo todos valores aceptables (Ruíz Bolívar, 2013).

Cuestionario de Regulación Emocional (ERQ) construida por Gross \& Thompson (2003) adaptado en el Perú por Gargurevich y Matos (ERQP, 2010), consta de dos subescalas, Reevaluación cognitiva y supresión emocional. Alcanza una confiabilidad Alpha de Cronbach para la subescala de reevaluación cognitiva de .72 y para supresión emocional de .74. En la Escala de regulación emocional se realizó el análisis factorial confirmatorio en el que se observan los dos factores.

La Confiabilidad, se obtuvo mediante consistencia interna a través de los coeficientes alfa y omega. Los valores en todas las variables en ambos coeficientes superan el 0.75 todos aceptables (Ruíz Bolívar, 2013).

\section{Procedimiento y Análisis de datos}

La aplicación de los instrumentos se llevó a cabo previa coordinación con docentes y autoridades de universidades públicas y privadas en días y horas que fueron programadas. La participación de los estudiantes fue anónima y voluntaria. Luego de la depuración de datos se procedió a verificar las propiedades psicométricas de los instrumentos previamente a la comprobación de hipótesis. La validez basada en la estructura interna se realizó mediante el análisis factorial confirmatorio (AFC) y la evaluación del modelo especificado se efectuó mediante el estimador de Mínimos Cuadrados Ponderados Robustos (WLSMV), dada la naturaleza ordinal de los ítems (Rigo y Donolo, 2018). Para el caso de la Escala de actitudes hacia el machismo fue necesario realizar el análisis factorial exploratorio (AFE) previo al AFC mediante el método Promax (rotación oblicua). La estimación de la fiabilidad fue mediante consistencia interna considerando las estructuras factoriales especificadas y en consideración de la naturaleza ordinal de los ítems, mediante el uso de los coeficientes Alfa y Omega, obtenidos por el Software Jamovi (Versión 1.1.9.0). Luego, se realizó la comprobación de la normalidad univariada de la distribución de los datos mediante la prueba de Shapiro Wilk. A partir de ello se decidió por la correlación de Spearman, dado que no hubo ajuste normal en ningún caso. Por último, en el análisis de comparación de grupos se verificó que los grupos tampoco mostraron ajuste normal, decidiéndose el uso de la U de Mann Whitney.

\section{RESULTADOS}

\section{Correlaciones entre las variables de estudio: Análisis Inferencial}

La normalidad de las variables se obtuvo con la prueba Shapiro Wilk para las variables en general y según sexo y tipo de universidad. En todos los casos se obtiene 
valores p (sig.) menores a 0.05 , no ajustándose a la distribución normal por lo que se usarán estadísticos no paramétricos.

\section{Tabla 1}

Prueba de normalidad para las variables estudiadas mediante la prueba de Shapiro Wilk

\begin{tabular}{lccc}
\hline & Shapiro Wilk & N & P \\
\hline Revaluación cognitiva & .980 & 830 & .000 \\
Supresión & .991 & 830 & .000 \\
Machismo general & .887 & 830 & .000 \\
A. hacia el dominio masculino & .761 & 830 & .000 \\
A frente a la superioridad masculina & .979 & 830 & .000 \\
A. frente a la socializ, del rol sexual M y F & .891 & 830 & .000 \\
A. frente al control de la sexualidad varón & .745 & 830 & .000 \\
A. hacia la dirección del hogar & .804 & 830 & .000 \\
A. hacia el papel paterno & .819 & 830 & .000 \\
\hline
\end{tabular}

Al inspeccionar la relación entre las actitudes hacia el machismo con las estrategias de regulación emocional en estudiantes universitarios según universidad de procedencia (pública y privada) de Lima Metropolitana, se evidencian en la muestra total correlaciones estadísticamente significativas $(\mathrm{p}<0.05)$ y directas entre la reevaluación cognitiva con el rechazo al machismo global, y en sus áreas dominio masculino, socialización del rol sexual, dirección del hogar y el papel paterno. Los evaluados que utilizan la reevaluación cognitiva, presentan mayor rechazo hacia el machismo global y en sus áreas dominio masculino, socialización del rol sexual, dirección del hogar y papel paterno. El uso de esta estrategia disminuye la experiencia afectiva negativa, al darse la reestructuración del significado de la emoción; dejando entrever participación igual en los diferentes contextos y en el rol paterno. Debido a que en ninguno de los casos el Rho llega a 0.30 (Ellis, 2010) tienen tamaños del efecto pequeños; tal como se aprecia en la Tabla 2.

De otro lado, existen correlaciones estadísticamente significativas $(\mathrm{p}<0.05)$ e inversas entre la estrategia supresión con el machismo total y en sus áreas dominio masculino, superioridad masculina, socialización del rol sexual, control de la sexualidad ejercida por los varones, dirección del hogar y el papel paterno. Los estudiantes que utilizan la supresión tienden a presentar menor rechazo hacia el machismo en general y en sus seis áreas. Si bien modifican la expresión de la respuesta emocional, pero no modifica la naturaleza de la emoción negativa (Gross, 1998) lo que es preocupante pues encierra aceptación a la superioridad masculina, y expresiones o admisión de violencia de género. Dado que el Rho es menor a 0.30 (Ellis, 2010), tienen tamaños del efecto pequeños. 
Tabla 2

Correlaciones entre estrategias de regulación emocional y machismo

\begin{tabular}{lcccccccc}
\hline & & $\begin{array}{c}\text { Machismo } \\
\text { general }\end{array}$ & $\begin{array}{c}\text { Dominio } \\
\text { Masculino }\end{array}$ & $\begin{array}{c}\text { Superiorid } \\
\text { masculina }\end{array}$ & $\begin{array}{c}\text { Socializ Rol } \\
\text { sexual }\end{array}$ & $\begin{array}{c}\text { Control } \\
\text { Sexual }\end{array}$ & $\begin{array}{c}\text { Direc. } \\
\text { hogar }\end{array}$ & $\begin{array}{c}\text { Papel } \\
\text { paterno }\end{array}$ \\
\hline Revaluación & $r_{s}$ &. $\mathbf{1 1 2}$ & $\mathbf{. 1 3 8}$ & .005 & $\mathbf{. 1 3 3}$ & .061 & $\mathbf{. 0 7 3}$ & $\mathbf{. 0 7 6}$ \\
cognitiva & $\mathrm{r}^{2}$ & $\mathbf{. 0 1 2}$ & $\mathbf{. 0 1 9}$ & .000 & $\mathbf{. 0 1 7}$ & .003 & $\mathbf{. 0 0 5}$ & $\mathbf{. 0 0 5}$ \\
& $\mathrm{p}$ & .001 & .000 & .885 & .000 & .078 & .035 & .028 \\
Supresión & $r$ & -.189 & -.182 & $\mathbf{- . 1 4 3}$ & $\mathbf{- . 1 2 3}$ & $\mathbf{- . 1 6 5}$ & $\mathbf{- . 1 6 3}$ & $\mathbf{- . 1 0 2}$ \\
& $\mathrm{r}^{2}$ & $\mathbf{. 0 3 5}$ & $\mathbf{. 0 3 3}$ & $\mathbf{. 0 2 0}$ & $\mathbf{. 0 1 5}$ & $\mathbf{. 0 2 7}$ & $\mathbf{. 0 2 6}$ & $\mathbf{. 0 1 0}$ \\
& $\mathrm{p}$ & .000 & .000 & .000 & .000 & .000 & .000 & .003 \\
& $\mathrm{~N}$ & 830 & 830 & 830 & 830 & 830 & 830 & 830 \\
\hline
\end{tabular}

Nota: $r=$ coeficiente de correlación; $r^{2}=$ tamaño del efecto; $p=$ nivel de significación

En la Tabla 3 al analizar las correlaciones entre las estrategias de regulación emocional con las actitudes hacia el machismo en sus seis áreas, en los varones se aprecia correlación estadísticamente significativa $(\mathrm{p}<0.05)$ y directa entre la reevaluación cognitiva con el rechazo al machismo solamente con el área dominio masculino; en el caso de las mujeres también existe similar correlación, adicionando rechazo al machismo total y en sus áreas de dominio masculino y socialización del rol sexual. Son ellas quienes asocian esta estrategia con mayor rechazo al machismo. Estos resultados nos indican que los hombres dan relevancia a la participación de la mujer en determinadas actividades, en cambio ellas consideran una intervención de manera igualitaria en los diferentes ámbitos, en la educación de los hijos y la expresión de afecto. Al alcanzar correlaciones Rho menor a 0.30 (Ellis, 2010) tiene un tamaño del efecto pequeño.

Con respecto a la supresión, los varones y mujeres evidencian correlaciones estadísticamente significativas $(\mathrm{p}<0.05)$ e inversas con el rechazo al machismo total, y en sus áreas dominio masculino ambos; superioridad masculina y socialización del rol sexual los hombres, y control de la sexualidad y la dirección del hogar ambos. Es decir, menor rechazo hacia el machismo. En el caso de los varones el uso de esta estrategia, que cambia la expresión de la respuesta emocional pero no el afecto negativo y estrés; asumen superioridad sobre la mujer en, aptitud, actividades laborales, crianza y cuidado de hijos, lo que puede traducirse en manifiesta actitud machista. Ello deja latente y en riesgo a las mujeres para acceder dominio del hombre en sus actividades y en su sexualidad; para aceptar ser controladas y el maltrato psicológico. Al obtener un Rho menor a 0.30 (Ellis, 2010) las correlaciones mantienen tamaños del efecto pequeños. 
Tabla 3

Correlaciones entre estrategias de regulación emocional y machismo en varones y mujeres

\begin{tabular}{|c|c|c|c|c|c|c|}
\hline & & & \multicolumn{2}{|c|}{ Reevaluación Cognitiva } & \multicolumn{2}{|c|}{ Supresión } \\
\hline & & & Varones & Mujeres & Hombres & Mujeres \\
\hline \multirow[t]{22}{*}{ Machismo } & Total & $\mathrm{r}$ & .096 & .106 & -.173 & -.117 \\
\hline & & $r^{2}$ & .009 & .011 & .029 & .013 \\
\hline & & $\mathrm{p}$ & .053 & .028 & .000 & .016 \\
\hline & Dominio Masculino & $r$ & .140 & .115 & -.152 & -.113 \\
\hline & & $r^{2}$ & .019 & .013 & .023 & .012 \\
\hline & & $\mathrm{p}$ & .005 & .018 & .002 & .020 \\
\hline & Super masculina & $r$ & -.009 & -.032 & -.158 & -.030 \\
\hline & & $r^{2}$ & .000 & .001 & .024 & .000 \\
\hline & & $\mathrm{p}$ & .850 & .506 & .001 & .544 \\
\hline & Socializ Rol sexual & $r$ & .093 & .138 & -.121 & -.089 \\
\hline & & $r^{2}$ & .008 & .019 & .014 & .007 \\
\hline & & $\mathrm{p}$ & .063 & .004 & .015 & .067 \\
\hline & Control Sexualidad & $r$ & .075 & .030 & -.125 & -.135 \\
\hline & & $r^{2}$ & .005 & .000 & .015 & .018 \\
\hline & & $\mathrm{p}$ & .134 & .542 & .012 & .005 \\
\hline & Direc,hogar & $\mathrm{r}$ & .061 & .072 & -.139 & -.125 \\
\hline & & $r^{2}$ & .003 & .005 & .019 & .015 \\
\hline & & $\mathrm{p}$ & .219 & .136 & .005 & .010 \\
\hline & Papel paterno & $\mathrm{r}$ & .090 & .053 & -.057 & -.071 \\
\hline & & $r^{2}$ & .008 & .002 & .003 & .005 \\
\hline & & $\mathrm{p}$ & .071 & .277 & .254 & .142 \\
\hline & & $\mathrm{N}$ & 405 & 425 & 405 & 425 \\
\hline
\end{tabular}

Nota: $r=$ coeficiente de correlación; $r^{2}=$ tamaño del efecto; $P=$ nivel de significación

En la tabla 4 se analiza la relación entre las áreas de las actitudes hacia el machismo con las estrategias de la regulación emocional según procedencia de universidad, los estudiantes de universidad pública; presentan correlaciones estadísticamente significativas $(\mathrm{p}<0.05)$ y directas entre la estrategia reevaluación cognitiva con el rechazo al machismo en general y en sus áreas de dominio masculino, socialización del rol sexual y el papel paterno. En los de privada, es directa, solo en socialización del rol sexual masculino y femenino. Ello evidencia que los de pública, que usan esta estrategia exhiben mayor rechazo hacia el machismo en general y en sus tres áreas; considerando la inclusión de la mujer en actividades 
comunes y en la educación de los hijos. A diferencia de los de privada que dan relevancia a una educación similar entre hombres y mujeres. Debido a que el Rho llega a 0.30 (Ellis, 2010) todas estas correlaciones mantienen tamaños del efecto pequeños.

Tabla 4

Correlaciones entre estrategias de regulación emocional y machismo en estudiantes de universidades públicas

\begin{tabular}{|c|c|c|c|c|c|c|}
\hline & & & \multicolumn{2}{|c|}{ Reevaluación Cognitiva } & \multicolumn{2}{|c|}{ Supresión } \\
\hline & & & Pública & Privada & Pública & Privada \\
\hline \multirow[t]{22}{*}{ Machismo } & Total & $\mathrm{r}$ & .116 & .121 & -.168 & -.253 \\
\hline & & $r^{2}$ & .013 & .014 & .028 & .064 \\
\hline & & $\mathrm{p}$ & .004 & .068 & .000 & .000 \\
\hline & Dominio Masculino & $r$ & -.151 & .127 & -.172 & --.205 \\
\hline & & $r^{2}$ & .022 & .016 & .029 & .042 \\
\hline & & $\mathrm{p}$ & .000 & .056 & .000 & .002 \\
\hline & Super masculina & $r$ & .036 & -.059 & -.138 & -.164 \\
\hline & & $r^{2}$ & .001 & .003 & .019 & .026 \\
\hline & & $\mathrm{p}$ & .380 & .375 & .001 & .013 \\
\hline & Socializ Rol sexual & $r$ & .122 & .157 & -.086 & -.209 \\
\hline & & $r^{2}$ & .014 & .024 & .007 & .043 \\
\hline & & $\mathrm{p}$ & .003 & .018 & .034 & .002 \\
\hline & Control Sexualidad & $r$ & .074 & .051 & -.158 & -.181 \\
\hline & & $r^{2}$ & .005 & .002 & .024 & .032 \\
\hline & & $\mathrm{p}$ & .071 & .446 & .000 & .006 \\
\hline & Direcci hogar & $\mathrm{r}$ & .069 & .099 & -.147 & -.209 \\
\hline & & $r^{2}$ & .004 & .009 & .021 & .043 \\
\hline & & $\mathrm{p}$ & .091 & .139 & .000 & .002 \\
\hline & Papel paterno & $\mathrm{r}$ & .081 & .109 & -.105 & -.103 \\
\hline & & $r^{2}$ & .006 & .011 & .011 & .010 \\
\hline & & $\mathrm{p}$ & .047 & .100 & .010 & .122 \\
\hline & & $N$ & 603 & 227 & 603 & 227 \\
\hline
\end{tabular}

En cuanto a la supresión ambos grupos, de pública y privada evidencian correlaciones estadísticamente significativas $(\mathrm{p}<0.05)$ e inversas con el rechazo al machismo total, y en sus áreas dominio masculino, superioridad masculina, socialización del rol sexual, control de la sexualidad, dirección del hogar y el papel paterno. El uso de esta estrategia por ambos muestra menor rechazo hacia el machismo total y en sus seis áreas, dejando escondido creer en un papel subordinado de la mujer en las actividades laborales, del hogar, manejo del hogar, en su sexualidad y una educación diferenciada por sexo; por ende, existe posibilidad de control 
a la mujer; pues esta estrategia regula la emoción cambiando la exteriorización de la respuesta emocional, pero no atenúa el estrés. Al obtener un Rho menor a 0.30 (Ellis, 2010) las correlaciones mantienen tamaños del efecto pequeños.

\section{DISCUSIÓN}

La violencia de género acentúa las diferencias entre el hombre y la mujer (Fernández-Alonso, 2003), modelos introyectados al interior de la familia, e inserta en los estándares sociales y culturales expresadas en actitudes machistas; acrecentado por la carencia de estrategias de regulación emocional.

El estudio muestra que la reevaluación cognitiva, se asocia positivamente con el rechazo al machismo global y en sus áreas dominio masculino, socialización del rol sexual, dirección del hogar y papel paterno; disminuyendo la experiencia afectiva negativa y aumentando la expresión emocional positiva, al reestructurar el significado, como lo plantean Gross (1998); Gross y John (2003) y Gross y Thompson (2007). Constituyéndose en factor de protección. Ello es diferente con la supresión, al ser la relación inversa, con el rechazo al machismo global y en sus seis áreas, lo que es preocupante, pues según Gross (1998) el usar esta estrategia, si bien modifica la expresión de la respuesta emocional, pero no cambia la emoción negativa, pasando a ser factor de riesgo, por la propensión a aceptar o expresar violencia de género y machismo invisible acorde a Castañeda (2009). Patterson \& Sanson (1999, citado en Esteves y Jiménez, 2017) y Huerta et al., (2019) revelaron que, vivir en ambientes de maltrato y falta de apoyo familiar, conllevan a pobre regulación de las emociones positivas como negativas, y afectación de la salud mental.

En los varones como en las mujeres, hay asociación baja entre la reevaluación cognitiva con rechazo al machismo, y en sus áreas dominio masculino y socialización del rol sexual en los hombres, como forma de aceptar una participación igualitaria. Ahora bien, la supresión es inversa en ambos, con rechazo al machismo y en cinco áreas. Al ocultar la expresión emocional puede dejar de manifiesto que el estar inmersos dentro de familias con patrones culturales machistas, estos se mantienen; afín con Oblitas (2009), manifestando que en el Perú los hombres presentan altos niveles de actitudes machistas; lo cual es un peligro para consentir el maltrato por ambos, según Castañeda (2009). Patterson \& Sanson (1999, citado en Esteves y Jiménez, 2017) revelan que, en jóvenes con maltrato en la relación de pareja, aumentan los trastornos de conducta y psicopatológicos A pesar de obtener un tamaño del efecto bajo, de acuerdo con Castañeda (2009) y Sanmartín (2006), se debe a la influencia de la concepción social y cultural y los modelos de familia peruana, asimismo estos hallazgos nos indican la importancia de tenerlos muy en cuenta. 
Según universidad, los de pública, al obtener asociación directa, entre reevaluación cognitiva y rechazo al machismo total y en sus seis áreas y, en los de privada solo en socialización del rol sexual conllevan a aceptar roles igualitarios en actividades comunes y en la educación de los hijos, al reformular el significado de la situación emocional y reducir el estrés. La relación inversa de la supresión con el rechazo al machismo en todas sus áreas por ambos grupos deja divisar la presencia de actitudes machistas como algo cotidiano, latente para el control y violencia. Giraldo (1972) señaló que los escenarios habituales, se hacen normales en hombres y mujeres. Park et al., (2012, citado en Souto, 2013) afirmaron que la etapa universitaria es básica para adquirir habilidades de autorregulación, al formar relación de pareja y afrontar las exigencias de la sociedad respecto a los roles de género.

Los hombres usan en mayor medida supresión, lo que deja oculto, a decir de Oblitas (2009) la expresión del machismo. Las mujeres presentan mayor rechazo al machismo. Los de universidad privada exhiben mayor rechazo al machismo global y en sus seis áreas en comparación con los de pública, Giraldo (1972) refuerza ello en razón a que el machismo es común a todos los estratos sociales y culturales.

El presente estudio permite observar la presencia de actitudes machistas en los estudiantes universitarios tanto en los de pública como privada lo cual nos muestra que sigue siendo un problema cultural en nuestro país arraigado a través de los modelos de familia que se trasmiten (Oblitas, 2009). Ello conlleva a plantear estudios que impliquen la aplicación de programas en los cuales se entrene en regulación emocional tanto a los hombres como a las mujeres, pues uno de los mayores retos es concientizar sobre las consecuencias de la violencia de género y así propiciar una formación integral de los estudiantes universitarios que no solo están insertos en los procesos de adaptación y cambio propios de la universidad sino también en los personales, interpersonales y de pareja.

Ahora bien, los resultados obtenidos en la presente investigación ameritan que la universidad como institución formadora asuma su rol a través de sus diferentes instancias, en la promoción y prevención, el diagnóstico y la intervención terapéutica de la población universitaria.

Dentro de las limitaciones del estudio, se observaron, falta de compromiso para viabilizar la aplicación de los instrumentos debido a trámites burocráticos en las universidades, los turnos y horarios que lentificaron la aplicación de los mismos.

\section{Conclusiones}

Los estudiantes universitarios de universidad pública como privada presentan correlación positiva y significativa entre la reevaluación cognitiva con el rechazo al 
machismo global y con sus áreas dominio masculino, socialización del rol sexual, dirección del hogar y el papel paterno. Con respecto a la supresión la correlación es inversa y significativa, si bien modifican la expresión de la respuesta emocional pero no modifica la naturaleza de la emoción negativa lo que incide en la propensión al machismo. Así mismo según sexo observamos similares correlaciones, en los hombres con el dominio masculino y, en las mujeres con el machismo total y las áreas de dominio masculino y socialización del rol sexual; considerando ambos una participación igualitaria. La supresión igualmente la correlación es inversa y significativa con menor rechazo al machismo total en ambos sexos. En las mujeres en dominio masculino, control de la sexualidad y dirección del hogar; y en los varones en dominio masculino, superioridad, socialización del rol sexual, control de la sexualidad y dirección del hogar. El uso de la supresión puede conllevar a presentar actitud machista y violencia de género.

Se encontraron, según universidad. en los de pública correlación positiva entre reevaluación cognitiva con rechazo al machismo global y con las áreas dominio masculino y socialización del rol sexual. En los de privada solo con socialización del rol sexual. En ambos grupos respecto a la supresión la relación con el rechazo al machismo es inversa y significativa, global y con todas las áreas del machismo. El uso de esta estrategia puede propiciar la posibilidad hacia actitudes machistas y a aceptar un papel subordinado de la mujer.

\section{Agradecimientos / Acknowledgments:}

Se agradece el apoyo brindado por las autoridades de las universidades públicas y privadas que participaron en este estudio.

\section{Fuentes de financiamiento / Funding:}

Financiado por la Universidad Nacional Mayor de San Marcos. Proyecto a través de Vicerrectorado de Investigación y Posgrado, con código A19180881, aprobado con Resolución Rectoral 03556-R-19

\section{Rol de los autores / Authors Roles:}

RHR: Responsable de la investigación.

RSO: Corresponsable de la investigación, adaptación de instrumentos.

NRS: Responsable del marco teórico.

JVG: Responsable de los antecedentes.

JEN: Responsable del Análisis de datos.

JVS: Responsable de recogida de datos. 
GCT: Aplicación de instrumentos y revisión de base de datos

AGH: Aplicación de instrumentos y revisión de base de datos

SMR: Responsable de la elaboración de la base de datos.

STQ: Adaptación de instrumentos y aplicación.

AGP: Administración de los instrumentos y procesamiento de datos.

JPM: Responsable de la recogida de datos.

AOM: Aplicación de instrumentos y revisión de base de datos

JQG: Adaptación de instrumentos y su aplicación.

\section{Aspectos éticos / legales; Ethics / legals:}

Los autores declaran no haber incurrido en aspectos antiéticos respetando los códigos de investigación con humanos, se solicitó la autorización respectiva y los participantes firmaron el consentimiento informado el cual brindaba datos de la naturaleza, medios y objetivos de la investigación.

\section{Conflicto de intereses / Competing interests:}

Los autores declaran no haber presentado conflicto de intereses en la investigación y elaboración del artículo.

\section{REFERENCIAS}

Ato, M., López, J. \& Benavente, A. (2013). Un sistema de clasificación de los diseños de investigación en psicología. Anales de Psicología, 3(29), 1038-1059. http://www. redalyc.org/pdf/167/16728244043.pdf

Bonino, L. (2004). Los Micromachismos. Revista La Cibeles, 2, 1-6. Retrieved from http://www.luisbonino.com/pdf/Los Micromachismos 2004.pdf

Brougham, R., Zail, C., Mendoza, C., y Miller, J. (2009). Stress, sex differences, and coping strategies among college students. Current Psychology, 28, 85-97.

Bustamante, M. (1990). Escala de actitudes hacia el machismo. Recuperado de http:// documents.mx/documents/escala-de-actitudes-hacia-el-machismo.html.

Calvo, G. y Camacho, R. (2014). La violencia de género: evolución, impacto y claves para su abordaje. Enferm Global. Vol. 13(33):424-39Castañeda, M (2007). El machismo invisible regresa. Mexico, DF. Taurus.

Castañeda, M. (2007). El machismo invisible regresa. Taurus. Mexico, DF.

Castañeda, M. (2009). El machismo invisible: un enfoque interpersonal. Recuperado de http://archivo.estepais.com/site/2009/el-machismo-invisibleun-enfoqueinterpersonal/ 
Ellis, P. (2010). The essential guide to effect sizes: Statistical power, meta-analysis, and the interpretation of research results. Cambridge, UK: Cambridge University Press.

Esteves, E. y Jimenez, T. (2017) Violencia en adolescentes y regulación emocional. España: International Journal of Developmental and Educational Psychology. INFAD Revista de Psicología, Nº1 - Monográfico 1, 2017. ISSN: 0214-9877. pp:97104. Recuperado en https://doi.org/10.17060/ijodaep.2017.n1.v2.922

Fernández Alonso, M.C. (2003). Violencia doméstica. Grupo de Salud Mental del Programa deActividades de Prevención y Promoción de la Salud (PAPPS) de la Sociedad Española de Medicina de Familia y Comunitaria. Ministerio de Sanidad y Consumo. Madrid. Recuperado de http://www.msc.es/ciudadanos/violencia/docs/ VIOLENCIA_DOMESTICA.pdf

Ferrando, P. y Anguiano-Carrasco, C. (2010). El análisis factorial como técnica de investigación en psicología. Papeles del psicólogo, 31(1), 18-33. https://www.redalyc. org/pdf/778/77812441003.pdf

Gargurevich, R. y Matos, L. (2010). Adaptación y estudio de las propiedades psicométricas del cuestionario de autorregulación emocional (ERQP). Revista de Psicología, UCV. Vol.: 12. (pp. 192 - 215).

Giraldo, O. (1972). El machismo como fenómeno psicocultural. Revista Latinoamericana de Psicología, vol. 4, núm. 3, pp. 295-309. Bogotá, Colombia

Gómez, H. (2007) La prevención de la violencia de género en adolescentes. Una experiencia en el ámbito educativo. Apuntes de Psicología. 25(3):325-340.

Gross, J.J. (1998). Antecedent- and response-focused emotion regulation: Divergent consequences for experience, expression and physiology. Journal of Personality and Social Psychology, 74, 224-237

Gross, J. J., \& John, O. P. (2003). Individual differences in two emotion regulation processes: Implications for affect, relationships, and well-being. Journal of Personality and Social Psychology, 85, 348-362.

Gross, J.J., \& Thompson, R.A. (2007). Emotion regulation: Conceptual foundations. In J.J. Gross (Ed.), Handbook of Emotion Regulation (pp. 3-24). New York: Guilford Press.

Hernández, R.; Fernández, C. y Baptista, P. (2014). Metodología de la Investigación, 6ta Edición México: Mc Graw Hill/Interamericana Editores S.A. de C.V.

Huerta, R., Santivañez, R., Escudero, J., Ramos, J., Pastor, S. (2019). Salud Mental y apoyo social percibido en escolares de cuarto grado de secundarias de Instituciones Públicas de la ciudad de Lima. Revista de Investigación en Psicología. Facultad de Psicología. UNMSM. 22(2), pp. 233-250. DOI: http://dx.doi.org/10.15381/rinvp.v22i2.17424

Instituto Nacional de Estadística e Informática (INEI, 2018). PERU Encuesta Demográfica y de Salud Familiar - Nacional y Regional" 2017. Recuperado en https://www.inei. gob.pe/media/MenuRecursivo/publicaciones_digitales/.../index.html 
Lopes, P. N., Salovey, P., Coté, S., y Beers, M. (2005). Emotion regulation abilities and the quality of social interaction. Emotion, 5, 113-118.

Oblitas, B. (2009). Machismo y violencia contra la mujer. Estudio de trabajo social. Universidad Nacional Mayor de San Marcos. Lima, Perú.

Rigo, D. y Donolo, D. (2018). Modelos de ecuaciones estructurales. Usos en investigación psicológica y educativa. Revista Interamericana de Psicología, 52 (3). 345 - 357. https://journal.sipsych.org/index.php/IJP/article/view/388

Ruíz-Bolívar, C. (2013). Instrumentos y técnicas de Investigación Educativa. (3 ${ }^{\mathrm{a}}$ ed.). Texas: DANAGA Training and Consulting.

Samaniego, E. y Freixas, A. (2010) Estudio sobre la identificación y vivencia de violencia en parejas adolescentes. Apuntes de Psicología. 28(3): 349-366

Sanmartín J. (2006) ¿Qué es esa cosa llamada violencia? Suplemento del Boletín Diario de Campo. 40:11-30.

Souto, A. (2013). Regulación emocional y estrés académico en estudiantes de fisioterapia. Tesis Doctoral. Coruña, España: Universidad da Coruña. https://ruc.udc.es/dspace/ bitstream/.../SoutoGestal_AntonioJose_TD\%20_2013.pdf?...4

Ventura-León, J. y Caycho-Rodríguez, T. (2017). El coeficiente Omega: un método alternativo para la estimación de la confiabilidad. Revista Latinoamericana de Ciencias Sociales, 15 (1). 625 - 627. https://www.redalyc.org/pdf/773/77349627039.pdf

Winters, J., Clift, R. y Dutton, D. (2004): An Exploratory Study of Emotional Intelligence and Domestic Abuse. Journal of Family Violence, 19 (5), 255-267. 